\section{The Ino80 complex prevents invasion of euchromatin into silent chromatin}

\author{
Yong Xue, ${ }^{1,3}$ Christopher Van, ${ }^{2,3}$ Suman K. Pradhan, ${ }^{1}$ \\ Trent $\mathrm{Su}^{1}{ }^{1}$ Jason Gehrke, ${ }^{1}$ Benjamin G. Kuryan, ${ }^{1}$ \\ Tasuku Kitada, ${ }^{1}$ Ajay Vashisht, ${ }^{1}$ Nancy Tran, ${ }^{1}$ \\ James Wohlschlegel, ${ }^{1}$ Craig L. Peterson, ${ }^{2,4}$ \\ Siavash K. Kurdistani, ${ }^{1,4}$ and Michael F. Carey ${ }^{1,4}$ \\ ${ }^{1}$ Department of Biological Chemistry, David Geffen School \\ of Medicine at University of California at Los Angeles, \\ Los Angeles, California 90095, USA; ${ }^{2}$ Program in Molecular \\ Medicine, University of Massachusetts Medical School, \\ Worcester, Massachusetts 01605, USA
}

Here we show that the Ino80 chromatin remodeling complex (Ino80C) directly prevents euchromatin from invading transcriptionally silent chromatin within intergenic regions and at the border of euchromatin and heterochromatin. Deletion of Ino80C subunits leads to increased H3K79 methylation and noncoding RNA polymerase II (Pol II) transcription centered at the Ino80C-binding sites. The effect of Ino80C is direct, as it blocks H3K79 methylation by Dot1 in vitro. Heterochromatin stimulates the binding of Ino80C in vitro and in vivo. Our data reveal that Ino80C serves as a general silencing complex that restricts transcription to gene units in euchromatin.

Supplemental material is available for this article.

Received November 20, 2014; revised version accepted January 16, 2015.

Euchromatin in the yeast Saccharomyces cerevisiae is characterized by Dot1-mediated H3K79 trimethylation (H3K79me3) (Briggs et al. 2002; van Leeuwen et al. 2002; Henry et al. 2003; Wood et al. 2003; Kao et al. 2004; Xiao et al. 2005; Pavri et al. 2006; Shilatifard 2006). H3K79me3, a mark closely associated with active transcription, is typically present in the coding regions of genes but absent from intergenic regions as well as subtelomeric heterochromatin ( $\mathrm{Ng}$ et al. 2003; Pokholok et al. 2005), both of which are transcriptionally silent. In heterochromatin, Sir3's BAH domain binds over H3K79 (Armache et al. 2011) and blocks access of Dot1 to its substrate (Altaf et al. 2007), but whether this is the only mechanism preventing euchromatin invasion into heterochromatin is not known. It has also been unclear what molecular factor delimits Dot 1 to transcribed regions and prevents silent regions flanking genes in euchromatin from being methylated at H3K79 and transcribed. Here

[Keywords: Ino80; Dot1; Sir proteins; heterochromatin; silencing; H3K79 methylation]

${ }^{3}$ These authors contributed equally to this work.

${ }^{4}$ These authors cosubmitted this work.

Corresponding author: mcarey@mednet.ucla.edu

Article is online at http://www.genesdev.org/cgi/doi/10.1101/gad.256255.114. we show that the Ino80 chromatin remodeling complex (Ino80C) demarcates units of transcription across the genome, confining gene activity to gene bodies and away from silent regions, including heterochromatin.

Ino80C is conserved from yeast to humans (for review, see Conaway and Conaway 2009) and slides mononucleosomes to a central position on a DNA template while tightly organizing nucleosomes within arrays (Udugama et al. 2011). Ino80C functions in maintaining genome stability and acts oppositely to the SWR-C complex by removing H2AZ from nucleosomes (Papamichos-Chronakis et al. 2006, 2011; Yen et al. 2013). Little is known of the function of Ino80C in gene regulation, but we argue that it is likely to be repressive, since it removes H2AZ, a histone variant associated with transcription (Conaway and Conaway 2009; Biterge and Schneider 2014). The EM structure and interaction map of Ino80C bound with a nucleosome show it to contain a core module bearing the Ino80 catalytic subunit, Rvb1/2, and Ies2; a module with Nhp10, Ies1, Ies3, and Ies5; and two chromatin docking modules bearing Arp8, Arp4, Taf14, and Ies4; and Arp5 and Ies6 (Tosi et al. 2013). Here we employed biochemistry, RNA-seq (RNA sequencing), and ChIP-seq (chromatin immunoprecipitation [ChIP] combined with deep sequencing) along with extensive informatics analysis to show that Ino80C demarcates units of transcription across the genome, confining gene activity to gene bodies and away from silent regions, including heterochromatin.

\section{Results and Discussion}

Ino80C is enriched at the boundaries of the transcribed region of a gene and prevents H3K79 methylation

To determine the genome-wide distribution of Ino80C, we performed ChIP-seq on the chromatin-binding subunit Arp5 and compared it with the positions of H3K4me3 and H3K79me3, histone modifications characteristic of active genes (Fig. 1 A-C). Figure 1A shows a genome-wide heat map, Figure $1 \mathrm{~B}$ shows a metagene profile, and Figure 1C shows a browser track of two typical genes. Collectively, the data illustrate that Ino80C binds upstream of the transcription start site (TSS) and downstream from the transcription termination site (TTS). Importantly, Ino80C flanks the peaks of H3K4 and H3K79 methylation, which, as expected, are enriched at the $5^{\prime}$ ends and the bodies of genes, respectively. Our positioning is somewhat consistent with previous studies, with the caveat that Ino80C is clearly upstream of both the TSS and H3K4 methylation, suggesting that it does not occupy the first nucleosome within the gene (Shimada et al. 2008; Yen et al. 2012).

The dense packing of genes in the $S$. cerevisiae genome raised the possibility that the Ino80C peaks could, in principle, be located upstream of the TSS, downstream from the TTS, or both. To distinguish between these, we plotted Arp5 upstream of the TSS of genes bearing di-

(c) 2015 Xue et al. This article is distributed exclusively by Cold Spring Harbor Laboratory Press for the first six months after the full-issue publication date (see http://genesdev.cshlp.org/site/misc/terms.xhtml). After six months, it is available under a Creative Commons License (Attribution-NonCommercial 4.0 International), as described at http:// creativecommons.org/licenses/by-nc/4.0/. 


\section{A}

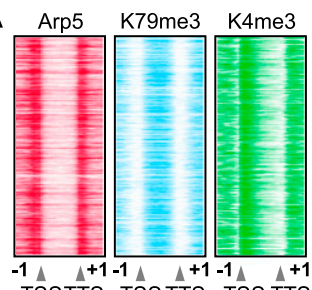

D TSSTTS TSSTTS TSS TTS

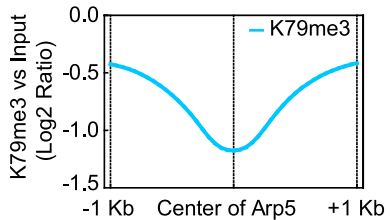

G

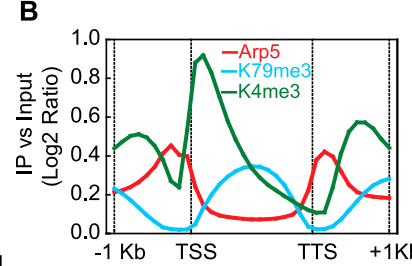

E

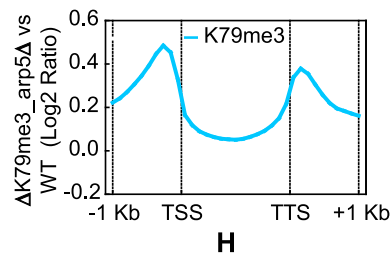

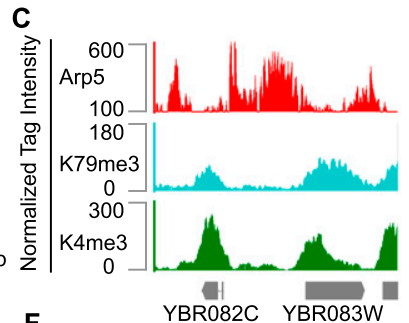

F

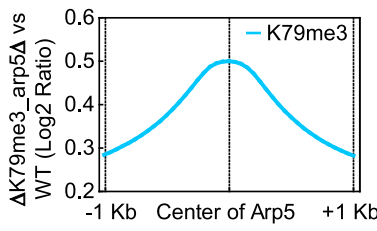

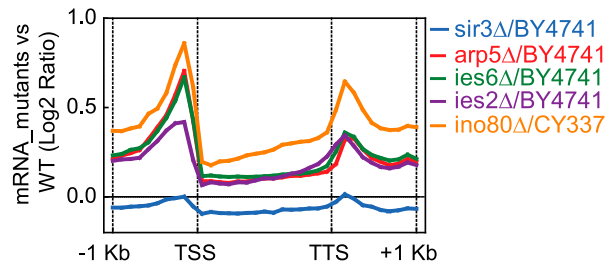

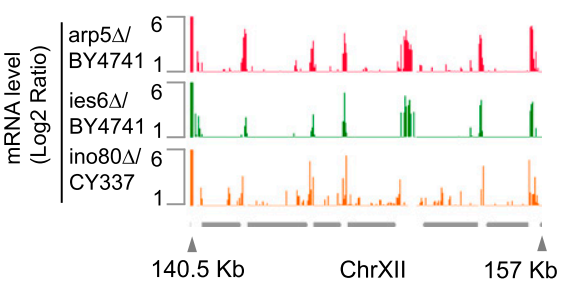

$140.5 \mathrm{~Kb} \quad$ ChrXII $\quad 157 \mathrm{~Kb}$

Figure 1. Ino80C is enriched at the boundaries of the gene body and anti-correlates with $\mathrm{H} 3 \mathrm{~K} 79$ methylation. (A) Distribution of Arp5, H3K79me3, and H3K4me3 across the gene through $1 \mathrm{~kb}$ upstream of the TSS to $1 \mathrm{~kb}$ downstream from the TTS at all genes. $\log _{2}$ ratios of immunoprecipitation (IP) versus input at significantly enriched windows were used, and each row represents one gene. (B) Average of metagene profiles of Arp5, H3K79me3, and H3K4me3 from A. (C) Patterns of Arp5, H3K79me3, and H3K4me3 at representative genes. (D) Average distribution of H3K79me3 around peaks of Arp5. $\log _{2}$ ratios of $\mathrm{H} 3 \mathrm{~K} 79 \mathrm{me} 3$ versus input at all windows are plotted. $(E)$ Metagene profiles of change of H3K79me3 in arp5s relative to wild type (WT) (BY4741). (F) Distribution of change of H3K79me3 in arp5s relative to wild type around the center of Arp5 peaks. (G) Metagene profiles of mRNA in Sir3 and Ino80 mutants alongside wild type. The $\log _{2}$ ratio of mRNA level in mutants versus wild type was plotted against the metagene. $(H)$ Patterns of mRNA $\left(\log _{2}\right.$ ratio) in Ino80 mutants versus wild type at chromosome XII 140,500-157,000.

vergent promoters and downstream from the TTS for convergent gene termini. The data in Supplemental Figure S1 confirm that Ino80C binds both upstream of the TSS and downstream from the TTS. Altogether, these data suggest that Ino80 physically bookends the gene unit, including the associated histone modifications, across the genome.

Our heat map and metagene analyses indicated that Arp5 binding and H3K79me3 might be mutually exclusive. Indeed, when we centered all Arp5 peaks and plotted levels of H3K79me3, we found that H3K79 methylation anti-correlates with Arp5 binding genome-wide (Fig. 1D). These data raised the possibility that Ino80C may be responsible for preventing access of the RNA polymerase II (Pol II) machinery and Dot1 to transcriptionally silent intergenic chromatin. To test this hypothesis, we analyzed H3K79 methylation in an arp5s strain and plotted the change in levels of methylation relative to wild-type cells. Figure 1E and Supplemental Figure S1C show that H3K79me3 increases in regions flanking genes in the arp5s strain, with little change within the gene body. Importantly, the increase in H3K79 methylation in arp54 occurred in the same location that Arp5 binds in the wildtype strains (Fig. 1F). In contrast, there was very little change in H3K4me3 in arp5s (Supplemental Fig. S1D). These data indicate that gene bookending by Ino80C restricts H3K79me3 to gene bodies.
Ino80C enhances transcriptional silencing in the flanking extragenic regions

Considering the strong correlation between $\mathrm{H} 3 \mathrm{~K} 79 \mathrm{me} 3$ and transcription (Im et al. 2003; Jaskelioff and Peterson 2003; Martin and Zhang 2005; Vakoc et al. 2006; Guenther et al. 2007; Kitada et al. 2012), we next asked whether enhanced H3K79 methylation in the flanking extragenic regions correlated with their ectopic transcription. We performed RNA-seq of polyA-containing transcripts (i.e., mRNA-seq) in wild-type and mutant strains of Ino80C bearing deletions of either ARP5, IES6, IES2, or INO80. Figure $1 \mathrm{G}$ shows a metagene analysis revealing that extragenic transcription occurred in all deletion mutants and peaked at the locations of Arp5 binding in wild-type strains. Figure $1 \mathrm{H}$ shows an example with a browser track. Although the transcript levels were typically much lower than in the gene body, they nonetheless were clearly apparent and statistically significant. Importantly, arp $5 \Delta$, ies24, ies64, and ino804 elicited similar increases in transcription, arguing that Ino80C and not a subcomplex is suppressing extragenic transcription.

To determine whether the extragenic transcription was due to sense and/or antisense transcription, we performed strand-specific mRNA sequencing. To exclude transcriptional signals from nearby genes, we analyzed only those genes that are separated from their neighboring genes on the opposite strand by $>1 \mathrm{~kb}$ either upstream of the TSS or downstream from the TTS. Supplemental Figure S1E shows that in the Ino80C mutants, both sense and antisense transcription are up-regulated. These data strongly argue that Ino80C prevents extragenic transcription associated with $\mathrm{H} 3 \mathrm{~K} 79 \mathrm{me} 3$. To investigate the detailed effect of arp5s mutants genome-wide, we clustered changes in sense and antisense transcription on Arp5-bound genes into three groups. As shown in Supplemental Figure S1F, we observed significant up-regulation of antisense transcription within transcribed regions in cluster 1 and outside of the transcribed region of genes in cluster 3 . Additionally, these genes also display up-regulated sense transcription both inside and adjacent to the gene. Overall, Ino80C prevents antisense and noncoding sense transcription in $>43 \%$ of genes in yeast (cluster 1 plus cluster 3 ).

\section{Ino80C prevents $\mathrm{H} 3 \mathrm{~K} 79$ methylation and enhances transcriptional silencing within heterochromatin in vivo}

Ino80C's role in suppressing H3K79 methylation and transcription within euchromatin raised the possibility that Ino80C may perform a similar role in heterochromatin. Analysis of Sir3 showed that, on average, its binding is highest in the region closest to the telomere, decreases 
gradually throughout the subtelomeric region, and reaches a minimum typically $\sim 20 \mathrm{~kb}$ from the telomere (Fig. 2A; Sperling and Grunstein 2009). Conversely, Arp5 binding increases as Sir3 binding decreases, indicating that Ino80C occupies the less dense heterochromatin adjacent to euchromatin. For example, Figure 2B shows a browser track of chromosome VIIL, where Ino80C binds poorly or not at all in dense heterochromatin near the telomere, but its binding becomes clearly apparent as the binding of Sir3 decreases.

On the basis of its transcriptional demarcation in euchromatin, Ino80C binding within heterochromatin suggested the possibility that it may function as a gatekeeper to prevent invasion of euchromatin into heterochromatin. Indeed, Figure 2C shows that, in the absence of Arp5, H3K79me3 increases throughout heterochromatin. Moreover, the greatest increase in H3K 79 me3 in an arp5s strain correlated with the average Arp5-binding peak observed in wild-type cells. Conversely, there was no measurable increase in subtelomeric levels of H3K4me3 (Supplemental Fig. S2).

Deletions of ARP5, IES6 (Fig. 2D), or INO80 (Fig. 2E) led to increased subtelomeric transcription similar to that observed in a strain bearing a deletion of SIR3 (Fig. 2D). Consequently, simultaneous deletions of SIR3 and ARP5 increased subtelomeric transcription in an additive manner (Fig. 2D). Interestingly, the enhanced transcription in the Ino80C mutants is greatest where the concentration of Sir3 decreases toward the euchromatic end of subtelomeric silent chromatin (Fig. 2F,G), and the effect of SIR3 deletion alone on transcription is greatest toward the telomeric end of subtelomeric silent chromatin. This point is illustrated clearly by plotting the increase in transcription in sir3s, arp $5 \Delta$, and ino804 strains on a browser track (Fig. 2G). These data suggest that Ino80C cooperates with the Sir complex to maintain heterochromatin gene silencing, especially in regions where the decreasing concentration of Sir3 may render it less effective in transcriptional silencing.

To determine whether this same silencing effect was observed at the Sir3-bound silent mating locus, we compared $H M L \alpha 1$ transcription in strains bearing deletions of Ino80C subunit genes and SIR3. Figure $2 \mathrm{H}$ shows a significant increase in transcription in all of the deletion mutants. We conclude that Ino80C and Sir3 contribute to transcriptional silencing in heterochromatin.

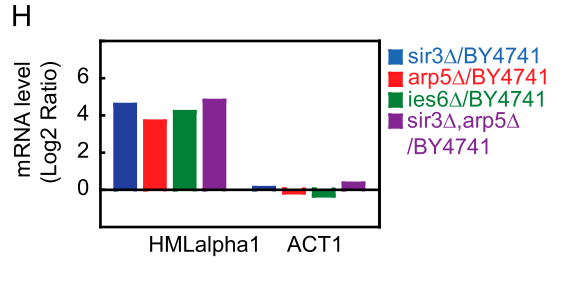

Figure 2. Ino80C is enriched in subtelomeric regions near the boundary of heterochromatin and euchromatin and affects silencing. (A) Average distribution of the Ino80C subunit Arp5 alongside Sir3 at subtelomeric regions of wild-type yeast. The moving averages of $\log _{2}$ Arp5 enrichment versus input (step size $=100$ base pairs [bp], window size $=40$ ) and $\log _{2}$ Sir3 enrichment relative to input $($ step size $=100 \mathrm{bp}$, window size $=20)$ were plotted according to distance from the telomere, from 0 to $20 \mathrm{~kb}$ (X-axis). (B) Distributions of Sir3 by $\mathrm{ChIP}$ array and of Ino80C subunit Arp5 by ChIP-seq at telomere region VIIL of wild-type yeast. $\log _{2}$ ratios of Arp5 and Sir3 enrichment versus input at all windows were used. $(C) \mathrm{H} 3 \mathrm{~K} 79 \mathrm{me} 3$ in wild type versus the arp5 $\Delta$ strain as a function of the distance from the telomere. The reads of wild type (WT) and the mutant versus input at all windows for the moving average of the $\log _{2}$ ratio (step size $=100 \mathrm{bp}$, window $=40$ ) were plotted according to distance from the telomere, from 0 to $40 \mathrm{~kb}(X$-axis). $(D)$ mRNA-seq analysis of Sir3 and Ino80 mutants alongside wild type. The moving average of $\log _{2}$ FPKM (fragments per kilobase of exon per million fragments mapped) (step size $=1$, window size $=100$ ) was plotted as a function of the distance from the telomere, up to $20 \mathrm{~kb}$. Wild-type yeast was compared with $\operatorname{sir} 3 \Delta$, arp $5 \Delta$, ies $2 \Delta$, and ies $6 \Delta$ strains. (E) Same as in $D$ except a strain bearing a deletion of the Ino80 catalytic subunit was employed. $(F)$ The ratio of mutant to wild-type expression as a function of the distance from the telomere. The mRNA of the mutant relative to wild type and the moving average of the $\log _{2}$ ratio (step size $=1$, window $=100)$ were plotted according to distance from the telomere, from 0 to $20 \mathrm{~kb}(X$-axis $)$. (G) Distributions of the $\log _{2}$ ratio of sir34, arp54, and ino $80 \Delta$ versus wild type according to distance from telomere VIIL, from 0 to $20 \mathrm{~kb}$. $(H)$ The effect of deletion mutants at $H M L \alpha 1$ on reversal of silencing is plotted as a bar graph.

\section{The Sir complex enhances Ino80C recruitment at heterochromatin in vivo and in vitro}

The overlapping functions of Ino80C and Sir3 in silencing within heterochromatin raised the key question of whether they also enforce each other's binding. To address this, we first asked whether Ino80C and Sir3 co-occupy genes within heterochromatin. Figure $3 \mathrm{~A}$ shows that within $20 \mathrm{~kb}$ of the telomere, 80 genes bind significant levels of both Sir3 and Arp5, including $H M L \alpha$ located in the subtelomeric region at the left end of chromosome III. To determine whether Sir3 affects Ino80C-mediated chromatin silencing, we performed locus-specific ChIP of Ino80C at $H M L \alpha$ in wild-type and sir3A strains. Loss of Sir3 significantly affected binding of Ino80C at the E and I silencer elements of $H M L$ but not at $A C T 1$, the control gene (Fig. 3B). These observations raised the 
possibility that the Sir complex may promote Ino80C binding.

To address this question, we purified Ino80C to near homogeneity via tandem affinity purification (TAP) and titrated it onto immobilized, unmodified chromatin either containing or lacking a fixed amount of purified Sir2, Sir3, and Sir4 proteins in vitro (Fig. 3C). Conversely, the Sir2, Sir3, and Sir4 proteins were titrated onto chromatin in either the absence or presence of a fixed amount of Ino80C (Fig. 3D). In both experiments, Ino80C was recruited to chromatin at higher levels in the presence of the Sir proteins. Our in vivo and in vitro data lead us to conclude that the Sir proteins contribute to Ino80C recruitment at heterochromatin.

\section{Ino80C blocks Dot1-mediated H3K79 methylation in vitro}

To determine whether Ino80C in turn directly prevents Dot1-mediated H3K79 methylation, we employed purified Ino80C and recombinant Dot1 in nucleosome modification assays. Figure 3, E and F, shows that Dot1 efficiently trimethylated H3K79 in the context of nucleosomes and free histone octamers. However, increasing amounts of Ino80C led to a dose-dependent inhibition of Dot1-mediated H3K79me3 on nucleosomes (Fig. 3E) but not on histone octamers (Fig. 3F). We conclude that Ino80C can directly block Dot1-mediated nucleosome methylation.

\section{Restricting gene expression to gene units}

Our data showing that Ino80C prevents H3K79 methylation outside of gene boundaries and silences transcription are consistent with the results on noncoding transcription in Ino80C mutants by the Buratowski and Tsukiyama laboratories (Alcid and Tsukiyama 2014; Marquardt et al. 2014). Importantly, we show that Ino80C is directly responsible for suppressing noncoding transcription within and around its binding site by blocking Dot1 and the Pol II machinery (Supplemental Fig. S3). The previously described cryptic unstable transcripts and stable unannotated transcripts (Xu et al. 2009) extensively overlapped with the Ino80Cbinding sites and were up-regulated in the Ino80C mutants (data not shown). This observation further supports the idea that Ino80C prevents noncoding transcription in silent extragenic regions.

Previous studies have shown that Ino80C is important for removal of $\mathrm{H} 2 \mathrm{AZ}$ incorporated either spuriously or as a dynamic process linked with gene activity (PapamichosChronakis et al. 2011; Yen et al. 2013). Thus, it is plausible that H2AZ insertion is among the processes blocked by Ino80C. Mutants in other chromatin remodeling complexes such as ISW1 and RSC activate divergent noncoding transcription (Marquardt et al. 2014). These complexes, however, do not typically colocalize with Ino80C, suggesting that they may operate independently by promoting regulated transcription and nucleosome stability within a gene (Yen et al. 2012). We do not know how Ino80 localizes to the flanks of genes. The localization of $\mathrm{H} 3 \mathrm{~K} 4 \mathrm{me} 3$ and H3K79me3 is directly linked with transcription, so perhaps such processes demarcate Ino80C outside of gene boundaries, where it serves to confine transcription to the gene-coding regions. It is plausible that ino80 also provides a crude insulator function to genes similar to that seen in higher eukaryotic genomes.
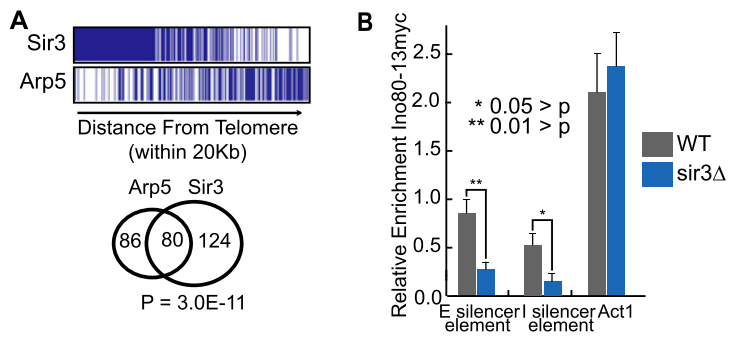

C Reconstituted chromatin

D Input Reconstituted chromatin

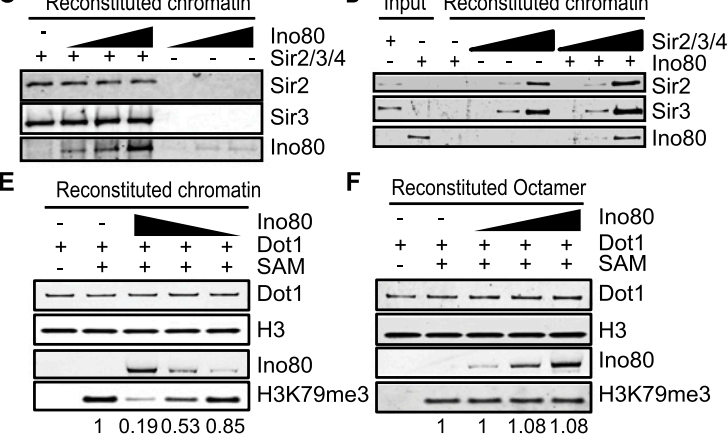

Figure 3. Mechanism of Ino80C silencing. (A) Overlap of Sir3 and Arp5 at telomere genes. Genes within $20 \mathrm{~kb}$ from the end of the chromosome are selected. The significant windows of $\log _{2}$ Arp5 enrichment versus input $>0.5$ and Sir3 enrichment versus input $>0.5$ were selected to overlap genes within $20 \mathrm{~kb}$ from the telomere, including a 100-bp extension around the gene body. The overlapping genes are considered Arp5- and Sir3-enriched. $(B)$ Targeted ChIP analysis of Ino80C in silent regions. ChIP and quantitative PCR (qPCR) were employed to compare Ino80-myc13 binding to the subtelomeric $H M L \alpha$ E and I silencer elements alongside a control ACT1 (Actin) gene in wild type and sir3 $4 .\left(^{\star}\right) P$-value $<0.05 ;\left(^{\star \star}\right) P$ value $<0.01$. (C) Immobilized template assay in which increasing amounts of Ino80 were incubated with $25 \mathrm{nM}$ immobilized chromatin in the presence and absence of $90 \mathrm{nM}$ Sir3 and $30 \mathrm{nM}$ Sir2/4. After washing, bound proteins were immunoblotted and scanned using an Odyssey imaging system. (D) Immobilized template assay in which 9.6 nM Ies1-TAP Ino80 complex in the presence of increasing amounts of Sir2, Sir3, and Sir4 and the chromatin was washed with buffer. The bound proteins were eluted and immunoblotted as above. $(E, F)$ The effect of Ino80C on H3K79 methylation by recombinant Dot 1 using a nucleosome $(E)$ or octamer $(F)$ as substrate. Recombinant His-Dot 1 was added to recombinant mononucleosomes or octamers in the presence of increasing molar ratios of Ino80C (Arp5-TAP). H3K79 methylation was measured by immunoblotting. The average ratio of three independent measurements of Ino80 inhibition of H3K79me3 by Dotl relative to no Ino80 is shown below the panel.

It is known that H4K16 acetylation prevents the Sir proteins from spreading from heterochromatin to euchromatin (Suka et al. 2002). However, the mechanism of preventing euchromatin from invading heterochromatin has been unclear. Our previous study showed that H3K79 methylation is a key feature of reversing silencing in subtelomeric regions (Kitada et al. 2012). We now demonstrate that heterochromatin can enhance Ino80C binding to prevent H3K79 methylation from invading into it. In principle, this binding works to enforce the silencing effect of the Sir complex within portions of heterochromatin where Sir3 levels are low. Collectively, these data suggest that Ino80C functions with the Sir proteins in some regions of heterochromatin to maintain silencing. Furthermore, Ino80C also directly blocks Dot 1 in vitro and in vivo at silent regions flanking genes. Taken together, our in vitro and in vivo data argue that Ino80C globally inhibits H3K79 
methylation and any of its causative influences or consequences.

\section{Materials and methods}

\section{Chromatin assembly and modification}

The 601-containing nucleosomal template was prepared as described (Kuryan et al. 2012). His-Dotl was purified using Talon beads and quantitated via Coomassie blue staining. Increasing molar ratios of HisDot1 and Ino80C were incubated for $1 \mathrm{~h}$ at $30^{\circ} \mathrm{C}$ (Kuryan et al. 2012), diluted into methylation reaction buffer $(10 \mathrm{mM}$ Tris- $\mathrm{Cl}$ at $\mathrm{pH} 8,5 \mathrm{mM}$ $\mathrm{MgCl} 2,50 \mathrm{mM} \mathrm{NaCl}, 0.1 \mathrm{mg} / \mathrm{mL}$ BSA, $1 \mathrm{mM} \mathrm{DTT}, 80 \mu \mathrm{M}$ S-adenosylmethionine), incubated for $1 \mathrm{~h}$, and immunoblotted for H3K79me3 (antibody from the Grunstein laboratory), total Histone H3 (ab1791), and TAP-tagged Arp5 and Dot1 (Sigma, H1029).

ChIP-PCR ChIP of Ino80-13myc was performed as described (Kitada et al. 2012) using anti-myc 9E10 from Millipore. Immunoprecipitated DNA was decross-linked overnight at $65^{\circ} \mathrm{C}$. Each ChIP experiment was performed on a minimum of three biological replicates. Real-time quantitative PCR (qPCR) was conducted using a Stratagene MX3000P thermal cycler and a SYBR Green qPCR mix with ROX using Roche FastStart Taq DNA polymerase. $P$-values were calculated by Student's $t$-test from three independent biological replicas.

\section{ChIP-seq}

Cells were synchronized in G2/M with nocodazole, released into $\alpha$ factor, and harvested at an OD of 0.6. Samples were cross-linked with formaldehyde, digested with micrococcal nuclease (Watanabe et al. 2013), and subjected to ChIP using an Arp5 antibody from Abcam (ab12099). ChIP was performed as described using antibodies against H3K79me3 (Kitada et al. 2012) and H3K4me3 (Active Motif, 39159). Libraries were prepared with a KAPA LTP kit and sequenced using the Illumina HiSeq 2000 platform. All sequenced reads were mapped to yeast genome version sacCer3 (SacCer_Apr2011) using bowtie 0.12.9 and default settings (Langmead et al. 2009). Next, Arp5, H3K79me3, and H3K4me3 were normalized to input using a custom script. The $S$. cerevisiae genome was divided into 50-base-pair (bp) windows, and significant windows with a $P$-value of $<0.001$ were selected as described (Ferrari et al. 2012). The $\log _{2}$ ratio of Arp5 versus input at significant windows was used to generate metagene profiles around the TSS and TTS. Unless specified, plots with $\log _{2}$ ratios of $\mathrm{H} 3 \mathrm{~K} 79 \mathrm{me} 3$ or $\mathrm{H} 3 \mathrm{~K} 4 \mathrm{me} 3$ versus input were generated using normalized raw reads from all windows. H3K 79 me3 was plotted against the Arp5-enriched region with Sitepro 0.6.6 (Shin et al. 2009).

\section{RNA-seq}

Libraries of mRNA were prepared with Illumina TruSeq RNA sample preparation kit version 2 or stranded RNA sample preparation kit. Libraries were sequenced, and reads aligned as above, excluding an identical region between $H M L$ and $H M R$, using TopHat 2.0 .8 with option -g 1 and $-\mathrm{N}$ 0 (Trapnell et al. 2009). Gene transcription levels were normalized to FPKM (fragments per kilobase of exon per million fragments mapped) using Cuffdiff 2.0.2 (Trapnell et al. 2010). For $\log _{2}$ ratio and log FPKM calculations, all transcripts with zero FPKM were replaced with 0.1. Mapped reads in the mutants were also normalized to reads in the wild type using a custom script. Because we were mapping changes in gene expression, windows without any overlapping reads in the wild-type cells were replaced with 0.1 for further treatment of the mutant expression patterns. The $\log _{2}$ ratios of mutants versus wild type at all windows were plotted against the distance from telomere ends or metagenes with a custom script. The data of BY4741, sir34, arp54, ies $6 \Delta$, and ies $2 \Delta$ are an average of two repeats. For strand-specific reads, the mapped plus or minus strand transcripts were separately plotted against genes on the minus or plus strand for metagene analysis. The sense transcription is the average of plus strand transcripts on plus strand genes and minus strand transcripts on minus strand genes. The antisense transcription is the average of plus strand transcripts on minus strand genes and minus strand transcripts on plus strand genes.

\section{Purification of yeast proteins}

Ino80C was purified from the TAP-tagged $S$. cerevisiae library as previously described ( $\mathrm{Li}$ et al. 2003). The Sir proteins were purified as described (Kitada et al. 2012)

\section{Immobilized template assay}

Immobilized template assays were performed as described (Kitada et al. 2012) using G5E4T assembled into chromatin (Lin et al. 2011; Kuryan et al. 2012) and incubated with either Ino80C or a mix of Sir3 and Sir2/4 in $25 \mu \mathrm{L}$ of binding buffer (100 mM KOAc, $20 \mathrm{mM}$ HEPES at $\mathrm{pH} 7.6,1 \mathrm{mM}$ EDTA, $10 \%$ glycerol) for $1 \mathrm{~h}$ at $30^{\circ} \mathrm{C}$ with a subsequent 45 -min incubation of the second protein. The beads were washed three times with $1 \mathrm{~mL}$ of binding buffer. Proteins were eluted in $200 \mu \mathrm{L}$ of $50 \mathrm{mM}$ Tris (pH 8.5) and $7 \mathrm{M}$ urea, immunoblotted, and scanned using an Odyssey imaging system.

\section{Accession numbers}

The Gene Expression Omnibus accession ID for aligned and raw data is GSE52000.

\section{Acknowledgments}

This work was supported by National Institutes of Heath grants R01 GM074701 and GM085002 to M.F.C., GM54096 to C.L.P., and CA178415 to S.K.K.

\section{References}

Alcid EA, Tsukiyama T. 2014. ATP-dependent chromatin remodeling shapes the long noncoding RNA landscape. Genes Dev 28: 2348-2360.

Altaf M, Utley RT, Lacoste N, Tan S, Briggs SD, Cote J. 2007. Interplay of chromatin modifiers on a short basic patch of histone $\mathrm{H} 4$ tail defines the boundary of telomeric heterochromatin. Mol Cell 28: 1002-1014.

Armache KJ, Garlick JD, Canzio D, Narlikar GJ, Kingston RE. 2011. Structural basis of silencing: Sir3 BAH domain in complex with a nucleosome at 3.0 A resolution. Science 334: 977-982.

Biterge B, Schneider R. 2014. Histone variants: key players of chromatin. Cell Tissue Res 356: 457-466.

Briggs SD, Xiao T, Sun ZW, Caldwell JA, Shabanowitz J, Hunt DF, Allis CD, Strahl BD. 2002. Gene silencing: trans-histone regulatory pathway in chromatin. Nature 418: 498.

Conaway RC, Conaway JW. 2009. The INO80 chromatin remodeling complex in transcription, replication and repair. Trends Biochem Sci 34: 71-77.

Ferrari R, Su T, Li B, Bonora G, Oberai A, Chan Y, Sasidharan R, Berk AJ, Pellegrini M, Kurdistani SK. 2012. Reorganization of the host epigenome by a viral oncogene. Genome Res 22: 1212-1221.

Guenther MG, Levine SS, Boyer LA, Jaenisch R, Young RA. 2007. A chromatin landmark and transcription initiation at most promoters in human cells. Cell 130: 77-88.

Henry KW, Wyce A, Lo WS, Duggan LJ, Emre NC, Kao CF, Pillus L, Shilatifard A, Osley MA, Berger SL. 2003. Transcriptional activation via sequential histone $\mathrm{H} 2 \mathrm{~B}$ ubiquitylation and deubiquitylation, mediated by SAGA-associated Ubp8. Genes Dev 17: 2648-2663.

Im H, Park C, Feng Q, Johnson KD, Kiekhaefer CM, Choi K, Zhang Y, Bresnick EH. 2003. Dynamic regulation of histone H3 methylated at lysine 79 within a tissue-specific chromatin domain. J Biol Chem 278: 18346-18352.

Jaskelioff M, Peterson CL. 2003. Chromatin and transcription: histones continue to make their marks. Nat Cell Biol 5: 395-399.

Kao CF, Hillyer C, Tsukuda T, Henry K, Berger S, Osley MA. 2004. Rad6 plays a role in transcriptional activation through ubiquitylation of histone H2B. Genes Dev 18: 184-195.

Kitada T, Kuryan BG, Tran NN, Song C, Xue Y, Carey M, Grunstein M. 2012. Mechanism for epigenetic variegation of gene expression at yeast telomeric heterochromatin. Genes Dev 26: 2443-2455.

Kuryan BG, Kim J, Tran NN, Lombardo SR, Venkatesh S, Workman JL, Carey M. 2012. Histone density is maintained during transcription mediated by the chromatin remodeler RSC and histone chaperone NAP1 in vitro. Proc Natl Acad Sci 109: 1931-1936.

Langmead B, Trapnell C, Pop M, Salzberg SL. 2009. Ultrafast and memory-efficient alignment of short DNA sequences to the human genome. Genome Biol 10: R25. 
Li B, Howe L, Anderson S, Yates JR III, Workman JL. 2003. The Set2 histone methyltransferase functions through the phosphorylated carboxyl-terminal domain of RNA polymerase II. J Biol Chem 278: 8897-8903.

Lin JJ, Lehmann LW, Bonora G, Sridharan R, Vashisht AA, Tran N, Plath K, Wohlschlegel JA, Carey M. 2011. Mediator coordinates PIC assembly with recruitment of CHD1. Genes Dev 25: 2198-2209.

Marquardt S, Escalante-Chong R, Pho N, Wang J, Churchman LS, Springer M, Buratowski S. 2014. A chromatin-based mechanism for limiting divergent noncoding transcription. Cell 157: 1712-1723.

Martin C, Zhang Y. 2005. The diverse functions of histone lysine methylation. Nat Rev Mol Cell Biol 6: 838-849.

Ng HH, Ciccone DN, Morshead KB, Oettinger MA, Struhl K. 2003. Lysine-79 of histone $\mathrm{H} 3$ is hypomethylated at silenced loci in yeast and mammalian cells: a potential mechanism for position-effect variegation. Proc Natl Acad Sci 100: 1820-1825.

Papamichos-Chronakis M, Krebs JE, Peterson CL. 2006. Interplay between Ino80 and Swrl chromatin remodeling enzymes regulates cell cycle checkpoint adaptation in response to DNA damage. Genes Dev 20: $2437-2449$.

Papamichos-Chronakis M, Watanabe S, Rando OJ, Peterson CL. 2011. Global regulation of H2A.Z localization by the INO80 chromatinremodeling enzyme is essential for genome integrity. Cell 144: 200-213.

Pavri R, Zhu B, Li G, Trojer P, Mandal S, Shilatifard A, Reinberg D. 2006. Histone $\mathrm{H} 2 \mathrm{~B}$ monoubiquitination functions cooperatively with FACT to regulate elongation by RNA polymerase II. Cell 125: 703-717.

Pokholok DK, Harbison CT, Levine S, Cole M, Hannett NM, Lee TI, Bell GW, Walker K, Rolfe PA, Herbolsheimer E, et al. 2005. Genome-wide map of nucleosome acetylation and methylation in yeast. Cell 122: 517-527.

Shilatifard A. 2006. Chromatin modifications by methylation and ubiquitination: implications in the regulation of gene expression. Annu Rev Biochem 75: 243-269.

Shimada K, Oma Y, Schleker T, Kugou K, Ohta K, Harata M, Gasser SM. 2008. Ino80 chromatin remodeling complex promotes recovery of stalled replication forks. Current Biol 18: 566-575.

Shin H, Liu T, Manrai AK, Liu XS. 2009. CEAS: cis-regulatory element annotation system. Bioinformatics 25: 2605-2606.

Sperling AS, Grunstein M. 2009. Histone H3 N-terminus regulates higher order structure of yeast heterochromatin. Proc Natl Acad Sci 106: 13153-13159.

Suka N, Luo K, Grunstein M. 2002. Sir2p and Sas2p opposingly regulate acetylation of yeast histone $\mathrm{H} 4$ lysine 16 and spreading of heterochromatin. Nat Genet 32: 378-383.

Tosi A, Haas C, Herzog F, Gilmozzi A, Berninghausen O, Ungewickell C, Gerhold CB, Lakomek K, Aebersold R, Beckmann R, et al. 2013. Structure and subunit topology of the INO80 chromatin remodeler and its nucleosome complex. Cell 154: 1207-1219.

Trapnell C, Pachter L, Salzberg SL. 2009. TopHat: discovering splice junctions with RNA-seq. Bioinformatics 25: 1105-1111.

Trapnell C, Williams BA, Pertea G, Mortazavi A, Kwan G, van Baren MJ, Salzberg SL, Wold BJ, Pachter L. 2010. Transcript assembly and quantification by RNA-seq reveals unannotated transcripts and isoform switching during cell differentiation. Nat Biotechnol 28: 511-515.

Udugama M, Sabri A, Bartholomew B. 2011. The INO80 ATP-dependent chromatin remodeling complex is a nucleosome spacing factor. Mol Cell Biol 31: 662-673.

Vakoc CR, Sachdeva MM, Wang H, Blobel GA. 2006. Profile of histone lysine methylation across transcribed mammalian chromatin. Mol Cell Biol 26: 9185-9195.

van Leeuwen F, Gafken PR, Gottschling DE. 2002. Dotlp modulates silencing in yeast by methylation of the nucleosome core. Cell 109: 745-756.

Watanabe S, Radman-Livaja M, Rando OJ, Peterson CL. 2013. A histone acetylation switch regulates H2A.Z deposition by the SWR-C remodeling enzyme. Science 340: 195-199.

Wood A, Krogan NJ, Dover J, Schneider J, Heidt J, Boateng MA, Dean K, Golshani A, Zhang Y, Greenblatt JF, et al. 2003. Bre1, an E3 ubiquitin ligase required for recruitment and substrate selection of Rad6 at a promoter. Mol Cell 11: 267-274.

Xiao T, Kao CF, Krogan NJ, Sun ZW, Greenblatt JF, Osley MA, Strahl BD. 2005. Histone $\mathrm{H} 2 \mathrm{~B}$ ubiquitylation is associated with elongating RNA polymerase II. Mol Cell Biol 25: 637-651.

Xu Z, Wei W, Gagneur J, Perocchi F, Clauder-Munster S, Camblong J, Guffanti E, Stutz F, Huber W, Steinmetz LM. 2009. Bidirectional promoters generate pervasive transcription in yeast. Nature 457: 1033-1037.
Yen K, Vinayachandran V, Batta K, Koerber RT, Pugh BF. 2012. Genomewide nucleosome specificity and directionality of chromatin remodelers. Cell 149: 1461-1473.

Yen K, Vinayachandran V, Pugh BF. 2013. SWR-C and INO80 chromatin remodelers recognize nucleosome-free regions near +1 nucleosomes. Cell 154: 1246-1256. 


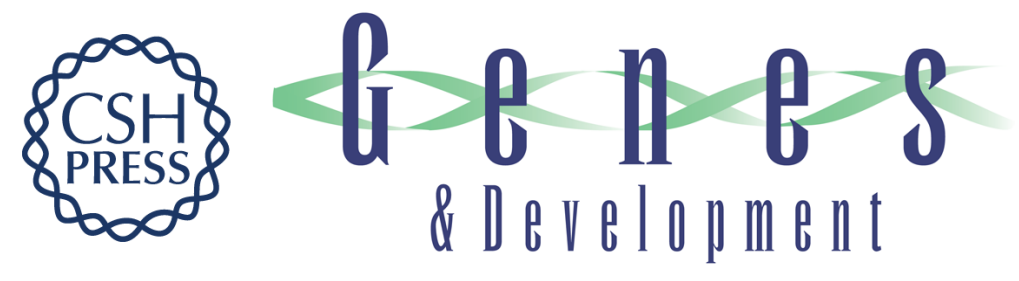

\section{The Ino80 complex prevents invasion of euchromatin into silent chromatin}

Yong Xue, Christopher Van, Suman K. Pradhan, et al.

Genes Dev. 2015, 29:

Access the most recent version at doi:10.1101/gad.256255.114

\section{Supplemental http://genesdev.cshlp.org/content/suppl/2015/02/17/29.4.350.DC1 Material}

References This article cites 41 articles, 16 of which can be accessed free at: http://genesdev.cshlp.org/content/29/4/350.full.html\#ref-list-1

Creative This article is distributed exclusively by Cold Spring Harbor Laboratory Press for the first Commons six months after the full-issue publication date (see

License http://genesdev.cshlp.org/site/misc/terms.xhtml). After six months, it is available under a Creative Commons License (Attribution-NonCommercial 4.0 International), as described at http://creativecommons.org/licenses/by-nc/4.0/.

Email Alerting Receive free email alerts when new articles cite this article - sign up in the box at the top Service right corner of the article or click here.

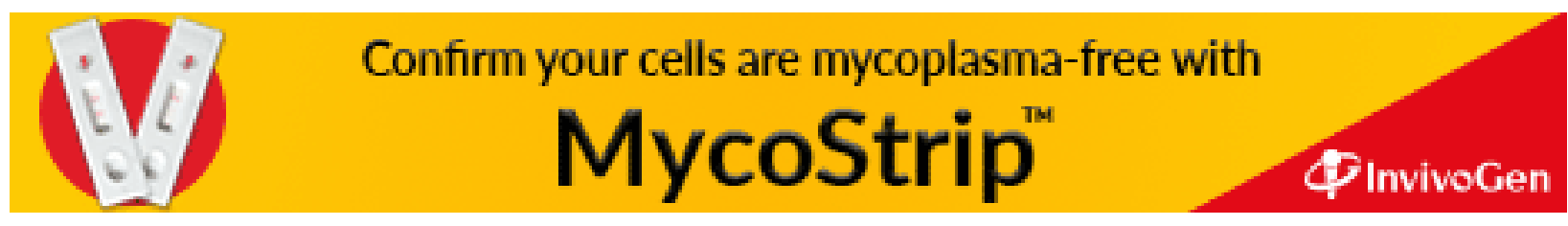

